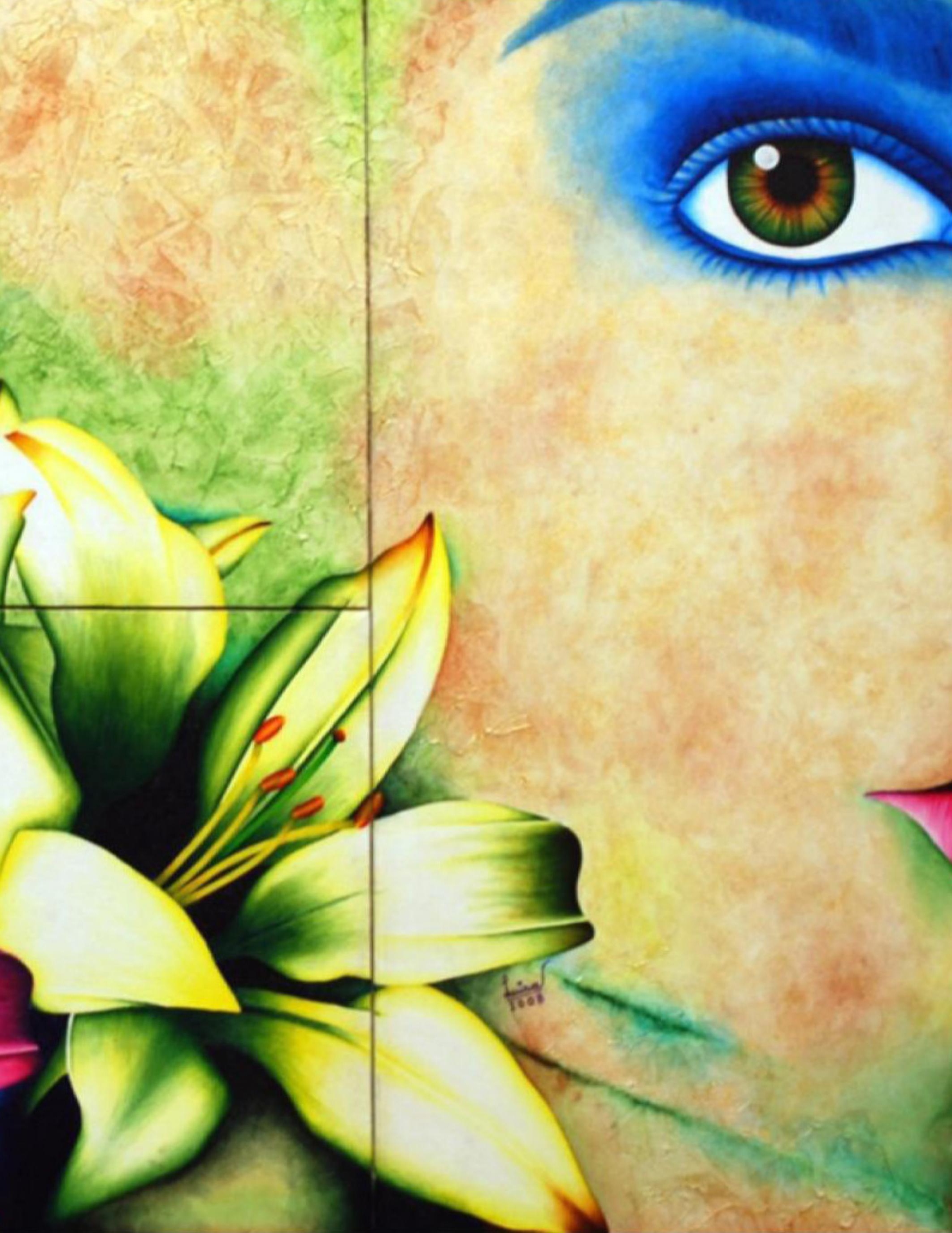




\section{La propuesta de un sistema unificado de medición estadística de indicadores de violencia contra las mujeres}

Martha Lorena Suazo*

RESUMEN. El objetivo principal de este artículo es presentar la propuesta de los indicadores de violencia contra las mujeres, que han consensuado instituciones públicas productoras de información estadística bajo la responsabilidad del Instituto Nacional de la Mujer, Corte Suprema de Justicia, Ministerio Público, Secretaría de Salud Pública, Secretaría de Seguridad, Instituto Nacional de Estadísticas y el Observatorio de Violencia de la Universidad Nacional Autónoma de Honduras. Se plantea la preocupación sobre la necesidad de poner en funcionamiento esta iniciativa, también se comenta sobre cómo relatan la violencia de género los indicadores, finalizando con una discusión sobre la necesidad que tiene el país de contar con herramientas de este tipo para la gestión de las políticas públicas.

Palabras clave: Indicador, género, violencia, políticas públicas

\section{LOS INDICADORES DEL SISTEMA DE VIOLENCIA CONTRA LAS MUJERES ${ }^{1}$}

\section{INTRODUCCIÓN}

La situación de violencia que vive el país, es una de las principales preocupaciones de la sociedad hondureña, exacerbado en la actualidad con el incremento de violencia contra las mujeres en todos los campos, conviertiendo la problemática en una prioridad de política pública, especialmente una prioridad de las políticas de género.
En ese aspecto, la implementación de un sistema unificado de medición estadística de indicadores de violencia contra las mujeres, requiere del compromiso y la capacitación del personal involucrado, tanto en el diseño como la recolección, tratamiento de la información y difusión de los resultados. Uno de los pasos importantes que han realizado las instituciones intervinientes, es el consenso en cuanto al marco conceptual que guiará el Sistema de Indicadores; se han suprimido los conceptos como femicidio/ feminicidio cuya conceptualización todavía se encuentra en debate, y se ha acordado utilizar el término de muerte violenta de las mujeres.

El objetivo principal de este artículo es presentar la propuesta de los indicadores de violencia contra las mujeres, que han consensuado instituciones públicas productoras de información estadística bajo, la responsabilidad del Instituto Nacional de la Mujer, Corte Suprema de Justicia, Ministerio Público, Secretaría de Salud Pública, Secretaría de Seguridad, Instituto Nacional de Estadísticas y el Observatorio de la Violencia de la Universidad Nacional Autónoma de Honduras, así como reflexionar sobre la necesidad de poner en funcionamiento esta iniciativa.

\section{II. ¿CÓMO RELATAN LA VIOLENCIA DE GÉNERO LOS INDICADORES?}

Para presentar lo que ocurre en el país con indicadores de violencia sensibles a la pregunta por género y

Recibido: septiembre 2009/ Aceptado y versión final: noviembre 2010.

*. Es Investigadora Social, Abogada con Maestría en Trabajo Social, tiene estudios de doctorado en Ciencias Sociales. Es Coordinadora de la Maestría en Demografía Social de la Universidad Nacional Autónoma de Honduras.

1. La fuente principal de este artículo es la Sistematización de la propuesta del Sistema Unificado de Medición Estadística de Indicadores de Violencia Contra las Mujeres elaborado por Martha Lorena Suazo en 2009. 
violencia, es necesario señalar que este tipo de abordajes se hace dentro de un contexto de investigación más amplio. En ese aspecto, es importante manifestar que en las estadísticas hondureñas la pregunta por la relación violencia y género es reciente, asimismo los indicadores sociales en general no siempre incluyen la perspectiva de género.

En cuanto al enfoque, las investigaciones de género a nivel de país han sido realizadas mayoritariamente desde un enfoque cualitativo, siendo muy pocos los casos en que el aspecto cuantitativo se incluye en el análisis, cuando se presentan datos más se hace en un sentido ilustrativo.

Teniendo en cuenta lo anterior, las instituciones que formulan indicadores sobre género y violencia en el país, deben estar sabidas y comprometidas debido a que los indicadores que se generan desde sus instituciones son insumos no sólo para las políticas públicas y para la investigación con perspectiva de género en general, sino también para poder tener abordajes instrumentales y empiristas.

La tarea está empezando, se puede decir que los estudios que en el país se han realizado para elaborar indicadores sociales sobre la relación de género y violencia son pocos; los primeros intentos por visibilizar estadísticamente el problema de la violencia en el país parten del Instituto Nacional de Estadísticas INE, institución que realizó un módulo de la Encuesta Permanente de Hogares de Propósitos Múltiples, con un capitulo adicional de violencia en el año 2003; y con la creación del Observatorio de la Violencia de la Universidad Nacional Autónoma de Honduras en el año 2005 se abrió la posibilidad de visibilizar y medir la magnitud de la violencia en Honduras de forma permanente, generando información sobre muertes violentas y lesiones.

\section{LA PROPUESTA DE UN SISTEMA DE INDICADO-} RES DE VIOLENCIA DE GÉNERO

La propuesta de un Sistema de Indicadores de Violencia Contra las Mujeres es el sistema unificado de medición estadística, en donde participan el Instituto Nacional de la Mujer, todas las instituciones del Sistema de Justicia productoras de estadísticas, la Secretaría de Salud, El Instituto Nacional de Estadísticas INE y el Observatorio de la Violencia; este instrumento está basado en conceptos y definiciones, que mayoritariamente tienen su fundamento en la legislación vigente que validan el indicador. Para consensuar las definiciones de los indicadores y la forma de cálculo, el personal técnico de estas instituciones recibió asesoría por técnicas especialistas de CELADE (Chile) y Eurosocial Justicia (España).

Los indicadores de violencia contra las mujeres, tienen como fuente de información las estadísticas de la Policía, Poder Judicial, Ministerio Público, Instituto Nacional de la Mujer y la Secretaría de Salud; se organizan en cinco categorías a saber:

1. Indicadores de búsqueda de justicia.

2. Indicadores de respuesta a situaciones de violencia.

3. Indicadores de seguimiento para medir incidencias y respuesta institucional.

4. Indicadores de salud, para medir incidencias y prevalencias.

5. Indicadores de respuesta institucional, atendiendo un mandato contenido en la Política Nacional de la Mujer en el eje de violencia.

Para la construcción de los indicadores se parte de la conceptualización del aspecto a medir, identificando las dimensiones que tiene, y cuando procede, las subdimensiones, a esos niveles se elaboran, uno o diversos indicadores por cada sub-dimensión. Con los indicadores de dimensión se compone un indicador único que incluye todas las medidas, y que en este sistema son tasas. A partir de la conceptualización de los indicadores se derivan los sistemas de indicadores que son un conjunto integrado de éstos.

\section{Dificultades}

Aunque parece sencillo, este emprendimiento primario se encuentra cruzado por una serie de complejidades, las definiciones de violencia contra las mujeres se 
encuentran contempladas en la legislación vigente y no existe posibilidad de reconocer otro tipo de violencia, como lo reconocen Belem do Pará y la Plataforma de Acción de Beijing.

Las instituciones participantes en el Sistema de Indicadores de Violencia contra las Mujeres, adolecen de debilidades en el campo de la gestión por diversos factores:

- Falta de continuidad, todavía las instancias productoras de estadísticas, carecen de un sistema estandarizado de captación de información, que impide el seguimiento.

- Falta de un sistema automatizado de captura de información, que provoca que en el camino se pierda información valiosa.

- Limitado presupuesto, que conspira contra la capacidad resolutiva.

- Recursos humanos con escasa o ninguna capacitación técnica especializada, para la generación de indicadores de género.

- Rotación del personal técnico, que vuelve lentos los procesos e inflan los presupuestos.

En el caso de algunas instituciones, como la Policía y la Secretaría de Salud, se presenta la dificultad de que son varias las unidades que procesan estadísticas, en ese aspecto, este esfuerzo no logra captar la totalidad de los indicadores que se producen.

Por acuerdo de las instituciones participantes: Poder Judicial, Ministerio Público, Policía, Secretaría de Salud, Observatorio de la Violencia/UNAH, El Instituto Nacional de Estadísticas INE y el Instituto Nacional de la Mujer INAM, se pensó que los indicadores de violencia contra las mujeres se incluirían como un apartado del Sistema de Indicadores Sociales en Niñez, Adolescencia y Mujer (SISNAM), sin embargo, este acuerdo no se concretó; actualmente INE, INAM se encuentran en un proceso tendiente a reactivar esta iniciativa.

Es así que un sistema de indicadores debe aportar información perdurable, periódica y rigurosa sobre el sistema social (Morales y López, 1993), bajo esa perspectiva, todavía no ha sido posible la publicación de los indicadores debido a que las instituciones no cuentan con series informativas, tampoco existe una estructura organizativa a nivel de las instituciones proveedoras de información que lidere este emprendimiento. ${ }^{3}$

\section{LOS INDICADORES DEL SISTEMA DE VIOLENCIA CONTRA LAS MUJERES}

\subsection{Indicadores de búsqueda de Justicia y de Res-} puesta a Situaciones de Violencia Contra las Mujeres

Los indicadores de violencia contra las mujeres que aporta el Poder Judicial, el Ministerio Público y la Policía son indicadores, cuantitativos, la unidad de medida son las tasas, los porcentajes, la periodicidad con que se reportan y se ingresan a la base de datos es mensual. Tienen su fundamento legal en la Ley Contra la Violencia Doméstica, la Constitución de la República, el Código Penal y los Tratados Internacionales relacionados, suscritos y ratificados por el país.

Los indicadores son proporcionados por el CEDIJ Centro Electrónico de Documentación e Información Judicial creado en el año 2003; esta unidad es la responsable de ordenar, analizar, clasificar, archivar, digitalizar, publicar y divulgar la información institucional.

Uno de los principios que orienta al CEDIJ es ubicar al Poder Judicial de Honduras entre las organizaciones de justicia que, en el ámbito internacional, presentan su información como un mecanismo de instauración del principio de transparencia.

$\mathrm{Al}$ integrarse en el CEDIJ la administración de la información, por medio de la fusión de las unidades que la manejaban en forma parcial, se da un enfoque integral y se promueve su utilización, ya que anteriormente no se había desarrollado una cultura del reporte y uso de la información, como mecanismo para visualizar el cumpli-

3. A Noviembre 2010, El INE junto con la comisión de Violencia del INAM están trabajando en reactivar esta iniciativa.

En el INE se está trabajando en una nueva página, la cual tiene un apartado de género y violencia, para la cual se están produciendo series informativas a partir del 2001. 
miento de la labor que puede ser verificada por la sociedad en su rol de auditoría de la función judicial, como parte de esta mejora se han ampliado las mediciones, a mediciones de género, edad y sexo. Las ciudades de San Pedro Sula y Tegucigalpa son las que aportan la mayor contribución de información a las bases de datos del CEDIJ.

El 19 de julio de 2002 se crea la Unidad Técnica de Reforma Penal, como un órgano adscrito a la Fiscalía General de la República, con el propósito de coordinar y centralizar todos los procesos de trabajo del sistema penal y ejecutar las políticas de modernización, en su estructura organizacional se encuentra la oficina de estadísticas.

La oficina de estadísticas cuenta con formatos estandarizados de captura de información a nivel nacional, que es ingresada a la base de datos de forma manual, utilizando el software Excel y Access. Las mediciones se hacen por género, edad y sexo; sin embargo, existen dificultades al consignar algunos datos, por ejemplo: la edad, y el estado civil. Las ciudades de Tegucigalpa y San Pedro Sula son las que más contribuyen con información. En el caso de la Policía esta cuenta con oficinas especializadas de captura de información

Se presenta a continuación la propuesta de indicadores del poder judicial, Ministerio Público y Policía (ver cuadro No. 1). Sobre la base de estos indicadores estas instituciones tienen capacidad de generar información estadística seriada, a partir del año 2007.

A manera de ejemplo se presenta la estructura y forma de cálculo de un indicador de búsqueda de justicia (Cuadro No. 2).

Cuadro No. 1. Indicadores de Búsqueda de justicia y de respuesta a Situaciones de Violencia Contra las Mujeres

\begin{tabular}{|c|c|c|c|}
\hline No. & $\begin{array}{l}\text { Indicadores del } \\
\text { Poder Judicial }\end{array}$ & $\begin{array}{l}\text { Indicadores del } \\
\text { Ministerio Público }\end{array}$ & $\begin{array}{l}\text { Indicadores de la } \\
\text { Policía }\end{array}$ \\
\hline 1 & Tipo de Violencia Declarada & Tasa de denuncias recibidas por violencia Doméstica & Mujeres víctimas de violencia doméstica \\
\hline 2 & Ingresos de Violencia Doméstica & $\begin{array}{l}\text { Tasa de denuncia por violencia doméstica por cada } 100 \text { mil } \\
\text { mujeres entre la edad de } 10 \text { y más de } 80 \text { años }\end{array}$ & Mujeres víctimas de violencia intrafamiliar \\
\hline 3 & Mecanismos de Protección Impuestos & $\begin{array}{l}\text { Medidas de seguridad por violencia doméstica impuestas } \\
\text { por la Fiscalía }\end{array}$ & Mujeres víctimas de delitos sexuales \\
\hline 4 & $\begin{array}{l}\text { Tasa de Resolución en Materia de Violencia } \\
\text { Doméstica }\end{array}$ & Audiencias con comparecencia de la Fiscalía & Mujeres víctimas de homicidios \\
\hline 5 & $\begin{array}{l}\text { Tasa de resolución en casos de violencia intra- } \\
\text { familiar y delitos Sexuales }\end{array}$ & $\begin{array}{l}\text { Sentencias dictadas por los Juzgados Contra la Violencia } \\
\text { Doméstica }\end{array}$ & Mujeres víctimas de acoso sexual \\
\hline 6 & Resoluciones por caducidad & $\begin{array}{l}\text { Sentencias con lugar en los Juzgados Contra la Violencia } \\
\text { Doméstica }\end{array}$ & Mujeres víctimas de violación \\
\hline 7 & Duración media de los procesos & $\begin{array}{l}\text { Sentencias dictadas sin lugar en los Juzgados Contra la } \\
\text { Violencia Doméstica }\end{array}$ & Mujeres víctimas de tentativa de violación \\
\hline 8 & $\begin{array}{l}\text { Población recluida por delitos de violencia } \\
\text { intrafamiliar y sexual }\end{array}$ & Orientación a usuarios por violencia doméstica & Mujeres víctimas de actos de lujuria \\
\hline 9 & $\begin{array}{l}\text { Población masculina recluida por delitos de } \\
\text { violencia intrafamiliar y sexual }\end{array}$ & Violencia doméstica en hombres & $\begin{array}{l}\text { Denuncias atendidas en la línea telefónica } \\
114 \text { en el Distrito Central }\end{array}$ \\
\hline 10 & $\begin{array}{l}\text { Beneficios otorgados a hombres recluidos por } \\
\text { delitos de violencia intrafamiliar y sexual }\end{array}$ & Síndrome de la mujer agredida & \\
\hline 11 & $\begin{array}{l}\text { Personas capacitadas en materia de violencia } \\
\text { doméstica }\end{array}$ & Homicidios en mujeres & \\
\hline 12 & Capacitaciones realizadas en materia de género & Homicidios en mujeres por cada 100 mil mujeres & \\
\hline 13 & & Mujeres lesionadas & \\
\hline 14 & & $\begin{array}{l}\text { Mujeres lesionadas por cada } 100 \text { mil mujeres según } \\
\text { población }\end{array}$ & \\
\hline 15 & & Suicidios en mujeres & \\
\hline 16 & & $\begin{array}{l}\text { Suicidios en mujeres por cada } 100 \text { mil mujeres en edad de } \\
10 \text { a } 44 \text { años de edad }\end{array}$ & \\
\hline 17 & & $\begin{array}{l}\text { Suicidios en mujeres entre la edad de } 60 \text { años y más por } \\
\text { cada } 100 \text { mil mujeres }\end{array}$ & \\
\hline
\end{tabular}

Fuente: CEDIJ, Unidad Estadística Ministerio Público, Policía. 
Cuadro No. 2. Indicador: Tipo de Violencia Declarada

\begin{tabular}{|l|l|l|l|}
\hline Definición: Es el porcentaje de resoluciones declaradas como violencia física, psicológica, sexual y patrimonial \\
\hline Unidad de Medida & Porcentaje \\
\hline Tipo de indicador & Cuantitativo \\
\hline Fuente & CSJ, Bases de datos de Violencia Doméstica, año 2007 \\
\hline Categorías (Sub- población): & Total nacional, tipo de violencia, sexo. \\
\hline Limitaciones & Desagregación por edad, base de datos de los Juzgados de Paz. \\
\hline Forma de cálculo & $\begin{array}{l}\text { Porcentaje del tipo de Violencia } \\
\text { declarada }\end{array}$ & Total de resoluciones de violencia física, psicológica, sexual y patrimonial & X100 \\
\hline
\end{tabular}

Fuente: elaboración propia en base indicadores elaborados por personal técnico de CEDIJ 2008.

\subsection{Indicadores de Seguimiento}

Los indicadores de violencia contra las mujeres utilizados en el Observatorio de la Violencia son indicadores cuantitativos, la unidad de medida es el porcentaje, la periodicidad con que se reportan y se ingresan a la base de datos es trimestral, semestral y anual, tienen como fuente de información las bases de datos de denuncia de la Policía Preventiva, la Dirección de Investigación Criminal, la Dirección de Medicina Forense y buscan homologar, validar y sistematizar información oficial. Tienen su fundamento legal en, la Constitución de la República, el Código Penal y los Tratados Internacionales relacionados suscritos y ratificados por el país.

El Observatorio $\mathrm{Na}$ cional de la Violencia y los tres observatorios locales, Distrito Central, San Pedro Sula y Santa Rosa de Copán, surgen en el marco del Programa Gobernabilidad y Democracia del PNUD por medio del Proyecto Armas Pequeñas, Seguridad y Justi- cia, teniendo como contraparte principal, la Universidad Nacional Autónoma de Honduras. El Observatorio para su funcionamiento y generación de información tiene la ventaja de contar con el presupuesto, los recursos técnicos y tecnológicos para un adecuado funcionamiento. En la captación de información estadística el Observatorio en ocasiones se encuentra con la dificultad de que las bases de datos de la clínica forense presentan atrasos en el procesamiento de la información, situación que se ha ido superando. El cuadro No. 3 presenta la nomenclatura de los indicadores de violencia de género que consensuó el observatorio para incorporar al Sistema de Indicadores y el cuadro No. 4 un ejemplo de la forma de cálculo de un indicador.

\section{Cuadro No. 3. Indicadores del Observatorio de la Violencia}

\begin{tabular}{|c|l|}
\hline No. & Indicador \\
\hline 1 & Mujeres muertas violentamente en un año a nivel nacional \\
\hline 2 & Mujeres muertas violentamente a nivel nacional en el contexto de relaciones afectivas o de convivencia \\
\hline 3 & Número de mujeres muertas en el área urbana \\
\hline 4 & Mujeres muertas en su casa \\
\hline 5 & Mujeres muertas por arma de fuego \\
\hline 6 & Rangos de edad de las mujeres muertas \\
\hline 7 & Ocupación principal de las mujeres muertas violentamente \\
\hline 8 & Estado civil de las mujeres muertas violentamente \\
\hline
\end{tabular}

\section{Cuadro No. 4. Indicador Mujeres Muertas Violentamente en un Año a Nivel Nacional}

\begin{tabular}{|l|l|l|l|}
\hline Definición: Es el número de mujeres muertas violentamente ocurridas a nivel nacional \\
\hline Unidad de Medida & Porcentaje \\
\hline Tipo de indicador & Cuantitativo \\
\hline Fuente & OV/UNAH/PNUD, Policía preventiva, DGIC- Medicina Forense \\
\hline Categorías (Sub- población): & OV/UNAH/PNUD, Policía preventiva, DGIC- Medicina Forense \\
\hline Limitaciones & ninguna & \\
\hline Forma de cálculo & $\begin{array}{l}\text { Mujeres muertas violentamente en un año } \\
\text { a nivel nacional }\end{array}$ & $=$ & Número de mujeres muertas violentamente (en un año) \\
\hline
\end{tabular}

Fuente: Indicadores elaborados por personal técnico del Observatorio de la Violencia 


\subsection{Indicadores de Respuesta Institucional}

\subsubsection{Indicadores del Instituto Nacional de la}

Mujer INAM. Los indicadores de violencia contra las mujeres utilizados por el Instituto Nacional de la Mujer, son indicadores cuantitativos, la unidad de medida es el número de casos, la periodicidad con que se reportan y se ingresan a la base de datos es anual, tienen su fundamento teórico en la Política Nacional de la Mujer Primer Plan Nacional de Igualdad de Oportunidades, Ley contra la Violencia Doméstica, el Código Penal, la Constitución de la República y los Tratados Internacionales relacionados ratificados por el país. El cuadro No. 5 presenta la nomenclatura de los indicadores propuestos y en el cuadro No. 6 a manera de ejemplo se presenta la forma de cálculo de un indicador.

\section{Tabla No.5. Indicadores del Instituto Nacional de la Mujer INAM}

\begin{tabular}{|l|l|}
\hline No. & Indicador \\
\hline 1 & Casa refugio a nivel nacional \\
\hline 2 & Capacitaciones a policías y otros \\
\hline 3 & $\begin{array}{l}\text { Número de cursos en materia de violencia de género, llevados a } \\
\text { cabo por INAM }\end{array}$ \\
\hline
\end{tabular}

Fuente: Unidad de Violencia INAM

Tabla No. 6. Indicador

\section{Casas refugio a Nivel Nacional}

\begin{tabular}{|c|c|}
\hline \multicolumn{2}{|c|}{$\begin{array}{l}\text { Definición: Centros de refugio para mujeres sobrevivientes de violencia } \\
\text { de género } \\
\text { *CAIM: Centro de Atención Integral para la Mujer }\end{array}$} \\
\hline Unidad de Medida & Número de Casas Refugio \\
\hline Tipo de indicador & Cuantitativo \\
\hline Fuente & INAM \\
\hline Categorías (Sub- población): & Total nacional \\
\hline Limitaciones & Ninguna \\
\hline Forma de cálculo & $\begin{array}{l}\text { Sumatoria de casas refugio por departa- } \\
\text { mento }\end{array}$ \\
\hline Periodicidad & Anual \\
\hline
\end{tabular}

Fuente: Indicadores elaborados por personal técnico del INAM.

\subsection{Indicadores de Salud}

Los indicadores de violencia contra las mujeres utilizados en la Secretaria de Salud, también son indicadores cuantitativos, siendo la unidad de medida el porcentaje, la periodicidad con que se reportan y se ingresan a la base de datos es mensual, tienen su fundamento teórico en la Ley contra la Violencia Doméstica, el Código Penal, la Constitución de la República y los Tratados Internacionales relacionados, ratificados por el país. En el cuadro No. 7 se puede apreciar la nomenclatura de los indicadores acordados por la Secretaría de Salud para ser ingresados al Sistema de Indicadores y a manera de ejemplo de la forma de cálculo de un indicador en el cuadro No. 8.

\section{Tabla No. 7. Indicadores de Salud}

\begin{tabular}{|l|l|}
\hline No. & Indicador \\
\hline 1 & Número de personas atendidas por violencia \\
\hline 2 & Personas que recibieron atención psicológica \\
\hline 3 & Hombres que recibieron educación \\
\hline 4 & Hombres que finalizaron el grupo de masculinidad \\
\hline 5 & Capacitaciones sobre violencia doméstica y violencia intrafamiliar \\
\hline 6 & Comités contra la violencia doméstica organizados \\
\hline 7 & Asesorías legales servidas \\
\hline 8 & Denuncias interpuestas \\
\hline 9 & Dictámenes elaborados por las Consejerías de Familia \\
\hline 10 & Acompañamiento a las víctimas \\
\hline
\end{tabular}

Fuente: Indicadores elaborados por personal de Secretaría de Salud.

Tabla No. 8. Indicador: Número de Personas Atendidas por Violencia

\begin{tabular}{|l|l|l|l|}
\hline \multicolumn{2}{|l|}{ Definición: Es el número de personas atendidas en las Consejerías de Familia } \\
\hline Unidad de Medida & Porcentaje \\
\hline Tipo de indicador & Cuantitativo & \multicolumn{2}{l|}{$\begin{array}{l}\text { Programa de Salud Mental, Conserjerías de Familia } \\
\text { y Secretaria de Salud }\end{array}$} \\
\hline Fuente & Sexo, edad, regiones departamentales \\
\hline $\begin{array}{l}\text { Categorías (Sub- } \\
\text { población): }\end{array}$ & ninguna & $\begin{array}{l}\text { Número de aten- } \\
\text { ciones por violencia } \\
\text { domestica }\end{array}$ \\
\hline Limitaciones & $\begin{array}{l}\text { Porcentaje de } \\
\text { personas aten- } \\
\text { didas en las } \\
\text { Consejerías de } \\
\text { familia. }\end{array}$ & $\begin{array}{l}\text { Total de población } \\
\text { de responsabilidad } \\
\text { programática }\end{array}$ & \\
\hline Forma de cálculo & X100 \\
\hline Periodicidad: Mensual
\end{tabular}

Fuente: Forma de cálculo elaborados por personal técnico de la Secretaría de Salud 


\section{A MODO DE CONCLUSIÓN}

A partir de lo presentado podemos decir que todavía no existe en el país una tradición en la construcción de indicadores asociados con género y violencia, hace falta la divulgación de los resultados obtenidos hasta ahora por parte de diferentes instituciones, en ese aspecto, la implementación del Sistema Unificado de Medición Estadística de Indicadores de Violencia de Género sería el canal idóneo para cubrir esa falencia.

Un Sistema Unificado de Medición Estadística de Indicadores de Violencia de Género se convertiría en un recurso ágil y oportuno para los tomadores de decisiones, así como en un recurso idóneo para la gestión de las políticas públicas.

Aunque instituciones como la Policía, el Ministerio Público, el Poder Judicial, la Secretaría de Salud, el Observatorio de la Violencia, han hecho esfuerzos por generar estadísticas desagregadas por sexo, no existe estandarización en las fuentes de datos, lo que impide la continuidad en las series, se hace necesario establecer un mecanismo de coordinación entre las instituciones productoras de indicadores de violencia de género que participan de la propuesta de un Sistema Unificado de Medición Estadística de Indicadores de Violencia de Género.

Estas limitaciones se refuerzan con el hecho de que aún hoy día, en el país existe poca producción y personal dedicado a los estudios de género cuanto menos los estudios relacionados con género y violencia, se puede afirmar que el tema no se encuentra dentro de las principales preocupaciones de las agendas de investigación institucionales y universitarias y consecuentemente de las instituciones financieras.

\section{GLOSARIO}

Audiencias con comparecencia de la fiscalía: Las audiencias se realizan con comparecencia del fiscal o apoderados legales. Ley de Violencia Doméstica artículo No. 4.
Caducidad: Lapso que produce la pérdida o extinción de una cosa o de un derecho/ Efecto que en el vigor de una norma legal o consuetudinaria produce el transcurso del tiempo sin aplicarlas, equiparándolas en cierto modo a una derogación tácita.

Casos ingresados: se refiere a los expedientes que llegan por primera vez a conocimiento del despacho.

Fuente: Manual de Indicadores del Poder Judicial de Honduras, año 2006.

Denuncia por violencia doméstica: Es mediante la cual una mujer victima de violencia física, psicológica, sexual o patrimonial la hace manifiesta en forma verbal o escrita en la estación de policía mas cercana, Ministerio Público o directamente en el Juzgado encargado de conocer los casos de violencia doméstica. Ley de Violencia Doméstica.

Escuela Judicial: es una unidad que forma y capacita a los (as) operadores (as) de justicia, apoyando las directrices de la Corte Suprema de Justicia en la detección de necesidades y programación de cursos de formación y capacitación en áreas específicas, en forma permanente, a nivel local y nacional; mediante un proceso académico integral, sistematizado y obligatorio, atendidos por profesionales altamente capacitados a nivel nacional e internacional, apoyados con el uso de metodologías participativas y tecnologías avanzadas.

Fuente: Manual de Indicadores del Poder Judicial de Honduras, año 2006.

Levantamiento de cadáveres: Es el examen del cadáver en el propio lugar del hecho o donde se ha encontrado el cuerpo con el propósito de recoger todos aquellos indicios capaces de permitir una fiel reconstrucción del hecho, reconocer la causa de la muerte y cómo se produjo ésta, así como vincular a los presuntos responsables. Ley de Autopsias Médica Obligatoria.

Hombres víctimas de Violencia Doméstica: son los que se creen agredidos o lesionados en su integridad física, psicológica, económica y patrimonial. Ley de Violencia Doméstica

Homicidios: dar muerte a una persona. Código Penal Código Procesal Penal 
Artículos 116, 119, 120, 121 del Código Penal;

Mecanismos de protección: son todas las medidas de seguridad, precautorias y cautelares aplicadas al imputado, para tutelar o restituir los derechos de las mujeres que sufren de violencia doméstica.

Medidas de Seguridad: son aquellas que persiguen evitar o detener la violencia en cualquiera de sus manifestaciones y prevenir males mayores. Con la sola Presentación de la denuncia, se impondrán de oficio, por el Juzgado competente.

Fuente: Ley Contra la Violencia Doméstica, Artículo 6, Numeral 1.

Medidas Precautorias: estas medidas se orientan a prevenir la reiteración de la violencia doméstica mediante la reeducación del denunciado y el fortalecimiento de la autoestima de la mujer.

Fuente: Ley Contra la Violencia Doméstica, Artículo 6, Numeral 2.

Medidas Cautelares: estas pretenden garantizar el cumplimiento de las responsabilidades familiares del denunciado y serán exclusivamente impuestas por el Juzgado o Tribunal competente, en los casos que le sean sometidos directamente o por remisión.

Fuente: Ley contra la Violencia Doméstica, Artículo 6, Numeral 3.

Medidas de seguridad: son aquellas que persiguen detener la violencia en cualquiera de sus manifestaciones y prevenir males mayores. Se aplicará por el juzgado o tribunal competente, con la sola presentación de la denuncia o de oficio y en casos urgentes, por el Ministerio Público o Policía.

Son medidas verbales o escritas, que serán aplicadas con un mínimo de 2 semanas y un máximo de 6 meses a criterio del Juez(a) o a solicitud de la mujer agredida en la presentación de la denuncia y en casos urgentes, por la policía o de los fiscales del Ministerio Público. Ley Contra la Violencia Doméstica, articulo 6 y 7.

Mujeres lesionadas: según el Código Penal Comete el delito de lesiones quien cause daños, que afecte el cuerpo o la salud física o mental de otra persona.

Código Penal, Código Procesal Penal, Ley Contra la
Violencia Doméstica

Artículos 138, 137, 133-A, 134, 135, 136, 133, del Código Penal.

Resolución: se refiere a los autos, fallos, o providencias de una autoridad judicial. Decisiones emitidas por un juez que pretende resolver una situación determinada.

Fuente: Manual de Indicadores del Poder Judicial de Honduras, año 2006.

Sentencia: decisión judicial que pone fin en la instancia al litigio civil o causa criminal, resolviendo los derechos de cada litigante o señalando la condenación o absolución del procesado.

Fuente: Manual de Indicadores del Poder Judicial de Honduras, año 2006.

Sentencias dictadas por los Juzgados Especiales Contra Violencia Doméstica: las sentencias dictadas se hacen en la primera audiencia o en la tercera audiencia ya en la lectura de la sentencia. Ley de Violencia Doméstica Artículo 7.

Sentencias con lugar en los Juzgados Contra la Violencia Doméstica. son aquellas donde se aplican las medidas cautelares que son dictadas solamente por el Juez.

Ejemplo:

-Pensión provisional de alimentos.

-Guarda y custodia.

-Acciones legales en materia de alimentos o formación de patrimonio familiar. Ley de violencia doméstica

Articulo 6 inciso 3.

Sentencias dictadas sin lugar en los Juzgados Contra la Violencia Doméstica. Cuando no se comprueba la denuncia presentada tanto por la mujer como por el hombre. Ley de Violencia Doméstica.

Suicidios: Quien intentare suicidarse, se le impondrá una medida de seguridad consistente en un adecuado tratamiento psiquiátrico. Código penal Artículo 124 del Código Penal.

Violencia Doméstica: todo patrón de conducta asociado a una situación de Ejercicio desigual de poder que se manifieste en el uso de la violencia física,

Psicológica, patrimonial, y/o económica y sexual; 
Ejercicio desigual de Poder: toda conducta dirigida a afectar, comprometer o limitar el libre desenvolvimiento de la personalidad de la mujer por razones de Género.

Fuente: Ley contra la Violencia Doméstica, Artículo 5, Numeral 1.

Violencia Física: Toda acción u omisión que produce un daño o menoscabo de la integridad corporal de la mujer, no tipificada como delito en el Código Penal.

Fuente: Ley Contra la Violencia Doméstica, Artículo 5, No 2.1.

Violencia intrafamiliar: quien emplee fuerza, intimidación o haga objeto de persecución a su cónyuge o ex cónyuge, a la persona con quien conviva o haya mantenido una relación concubinario o aquella que haya procreado un hijo con la finalidad de causarle daño físico o emocional o para dañar sus bienes y descendientes. Código Penal

Código Procesal Penal

Constitución de la República de Honduras

Convenciones

Ley de Igualdad de oportunidades

Artículos 179-A, 179-B del Código Penal; 26 Código Procesal Penal Artículo 59 y 68 de la Constitución de la República.

Usuario víctimas de violencia doméstica. Usuario es la persona que es víctima de violencia doméstica e interpone la denuncia sea esta hombre o mujer. Ley de Violencia Doméstica, artículo 9.

Violencia Sexual: toda conducta que entrañe amenaza o intimidación que afecte la integridad o la autodeterminación sexual de la mujer, tal como las relaciones sexuales no deseadas, la negación a anticoncepción y protección, entre otras, siempre que dichas acciones no se encuentren tipificadas como delito en el Código Penal.

Fuente: Ley Contra la Violencia Doméstica, Artículo 5, No 2.3.

\section{BIBLIOGRAFÍA}

Congreso Nacional de la República de Honduras, 1982. Constitución de la República, Decreto Legislativo No de 131 del 11 de Enero de 1982. Tegucigalpa, Honduras.

Congreso de la República de Honduras, 1998. Ley Contra la Violencia Doméstica, Decreto Legislativo No 132-97 de 15 de Noviembre de 1997. Tegucigalpa, Honduras.

Congreso de la República de Honduras, 2000. Ley de Igualdad de Oportunidades, Decreto Legislativo No 34-2000 de 28 de abril del 2000. Tegucigalpa, Honduras.

Congreso de la República de Honduras, 1983. Código Penal, 26 de Septiembre de 1983. Tegucigalpa, Honduras.

Congreso de la República de Honduras, 1984, Código de Familia Decreto No 76-84, 1984. Tegucigalpa, Honduras.

Congreso de la República de Honduras, 1996. Código de la Niñez y la Adolescencia, Decreto No. 73-96 del 5 de Septiembre de 1996. Tegucigalpa, Honduras.

INE, 2004, Encuesta Permanente de Hogares de Propósitos Múltiples, Módulo de Violencia. Septiembre 2003, Tegucigalpa, Honduras.

INAM, 2001, Política Nacional de la Mujer, Primer Plan Nacional de Igualdad de Oportunidades. 2002-2007. Tegucigalpa, Honduras.

Morales, J. F. y M. López (1993), "Bases para la construcción de un sistema de indicadores sociales de estereotipia de género", en Psicothema, Oviedo, Facultad de Psicología de la Universidad de Oviedo/Colegio Oficial de Psicólogos del Principado de Asturias, vol. 5, núm. suplemento, pp. 123-132.

Suazo Matute Martha Lorena, 2009 Sistematización del Sistema Unificado de Medición Estadística de Indicadores de Violencia Contra Las Mujeres, Instituto Nacional de la Mujer INAM, Poder Judicial, Instituto Nacional de Estadísticas. 